\title{
Compact acoustic sensor based on air-backed mandrel coiled with optical microfiber
}

\author{
George Y. Chen, ${ }^{*}$ Gilberto Brambilla, and Trevor P. Newson \\ Optoelectronics Research Centre, University of Southampton, Southampton SO17 1BJ, UK \\ ${ }^{*}$ Corresponding author: gyc1g09@orc.soton.ac.uk
}

We propose and demonstrate a compact acoustic sensor comprising a $2 \mu \mathrm{m}$ diam, $35 \mathrm{~mm}$ length optical microfiber coiled around a $3 \mathrm{~mm}$ diam air-backed mandrel. Acoustic waves induce local pressure variations that change the mandrel diameter and thus the optical path length of the mode propagating in the microfiber. The phase modulation is detected via a single-fiber polarimetric interferometer. The low stiffness and minimum bend radii of microfibers gives rise to extremely small package sizes without compromising acoustic responsivity.

Acoustic sensing, with its commercial use spanning several decades, is one of the most successful applications in the field of optical fiber sensors. Rapid advances in this area have been driven by the increasing demand from industries, such as defense, most notably for hydrophones. The acoustic signatures of tanks, aircrafts, helicopters, and submarines can be monitored for high-precision battlefield awareness and surveillance. Optical acoustic sensors in the form of an air-backed mandrel (ABM) have proven to be a simple yet effective design with the potential for very high acoustic responsivity [ $\underline{1}-\underline{3}]$. Scaling down the size of acoustic sensors gives a battlefield advantage due to easier deployment and disguise in all types of terrain. However, due to the minimum bend radii of standard optical fibers, very compact designs are not feasible. Even though state-of-the-art bend-insensitive fibers can achieve a bend radius down to several millimeters [4], the fiber diameters are typically very large relative to the wall thickness of the downscaled transducer, leading to severe limitations on the acoustic responsivity.

In this Letter, we present the first experimental demonstration of a compact ABM exploiting the excellent mechanical properties of optical microfiber $(\mathrm{OM})$ for acoustic sensing in air. Light in an $O M$ is guided by the large refractive index contrast between the silica core and air cladding. Due to the large numerical aperture, bending radii of a few micrometers can be readily achieved with relatively low bending loss []ㅡ.

The operating principle of the ABM is illustrated in Fig. $\underline{1(\mathrm{a})}$. Acoustic waves produce local pressure variations that uniformly deform the hollow mandrel and exert strain on the coiled OM. The change in optical path length translates into a phase modulation that is detected using a single-fiber polarimetric interferometer, which reduces design complexity and facilitates better immunity against environmental perturbations. The upper measurable limit of acoustic pressure is governed by the clearance between the silicone rubber and the stainless steel rod.

The sensor was built in two main stages. First, the hollow mandrel was fabricated from silicone rubber chosen for its low Young's modulus. A relatively thick wall was chosen to ensure a high resonant frequency, which yields a wider operating bandwidth in the linear regime. A double-headed stainless steel pin was inserted in the mandrel as a rigid support. The two ends of the mandrel were epoxy-sealed with high-density polyethylene caps. Figure $\underline{1(b)}$ shows the dimensions of the ABM, which only weighs $\sim 0.5 \mathrm{~g}$. For the second stage, a length of standard telecom single-mode fiber (SMF-28) was heated and stretched using a stationary ceramic heater and a pair of automated translation stages [6]. The tapering process lasted 
$\sim 10$ min with an initial stretching speed of $1 \mathrm{~mm} / \mathrm{s}$ that was gradually slowed to ensure an adiabatic taper profile. The resulting OM had a uniform waist of $2 \mu \mathrm{m}$ diam and $35 \mathrm{~mm}$ length. A thin layer of low refractive-index polymer (Efiron UV-373, $n=1.373$ ) was deposited on the mandrel before and after embedding the OM to en-sure good confinement of light. The OM was machine-wound 3.5 turns around the mandrel waist with a large pitch $(>0.5 \mathrm{~mm})$ to prevent coupling between adjacent turns. Ultraviolet curing of the polymer coating ensured geometric stability, efficient transfer of forces, and ro-bustness from external effects. The optical loss of the fabricated sample was $\sim 6 \mathrm{~dB}$ at the wavelength $\lambda=1556 \mathrm{~nm}$, which can be attributed to Rayleigh scattering due to microbubbles and particles in the polymer.

(a)
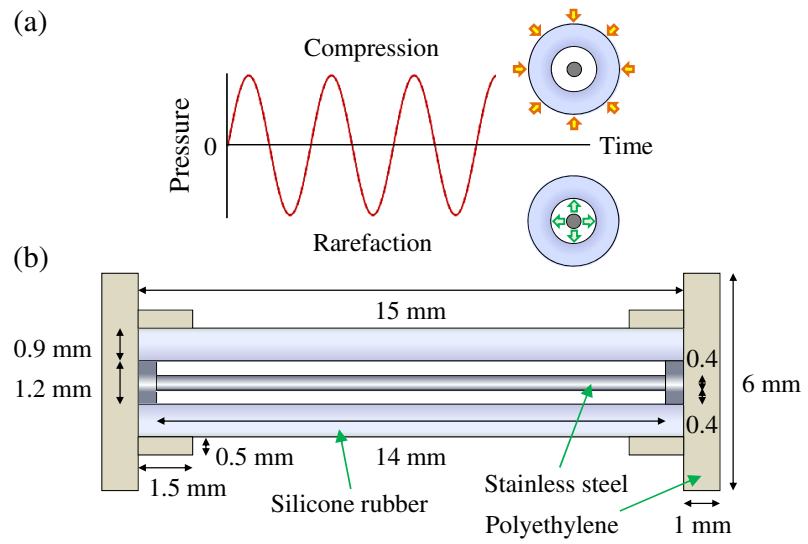

Fig. 1. (Color online) (a) Working principle of the ABM and (b) a schematic.

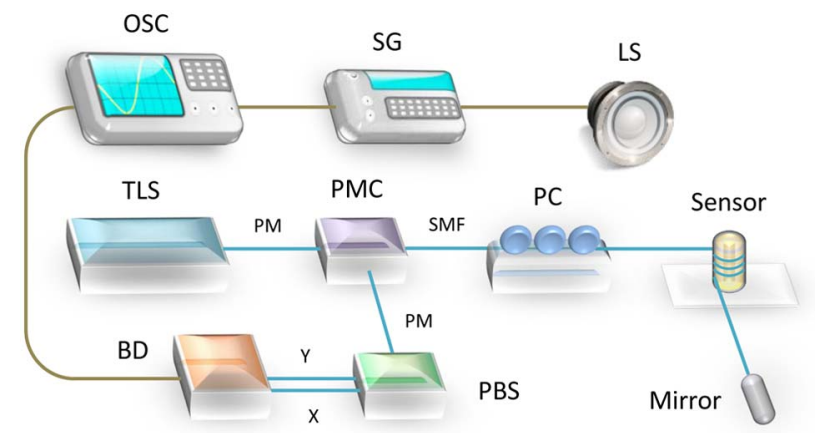

Fig. 2. (Color online) Schematic of the experimental setup. TLS, tunable laser source; PMC, polarization-maintaining coupler; PC, polarization controller; BD, balanced detector; PBS, polarization beam splitter; OSC, digital oscilloscope; SG, signal generator; LS, loudspeaker with power amplifier; PM, polarization-maintaining fiber; SMF, single-mode fiber.

The setup shown in Fig. 2 was used to test the sensor. Linearly polarized light at $\lambda 1556 \mathrm{~nm}$ from a tunable laser source (TLS) was split into two orthogonal axes due to the axes of the birefringent OM not being aligned with those of the incident beam. The differential phase of the two eigenmodes varies as a function of the acoustic pressure. A mirror was employed to double the differential phase and thus the responsivity of the sensor. The returning light combined interferometrically at the splice between the polarization-maintaining coupler (PMC) and the SMF, before the polarization beam splitter (PBS) separated the two polarizations for signal pro-cessing in the balanced detector (BD). The AC-coupled oscilloscope (OSC) captured 1000 data points per measurement with two measurement averages. The acoustic pressure at the location of the sensor was measured using a calibrated commercial microphone. 


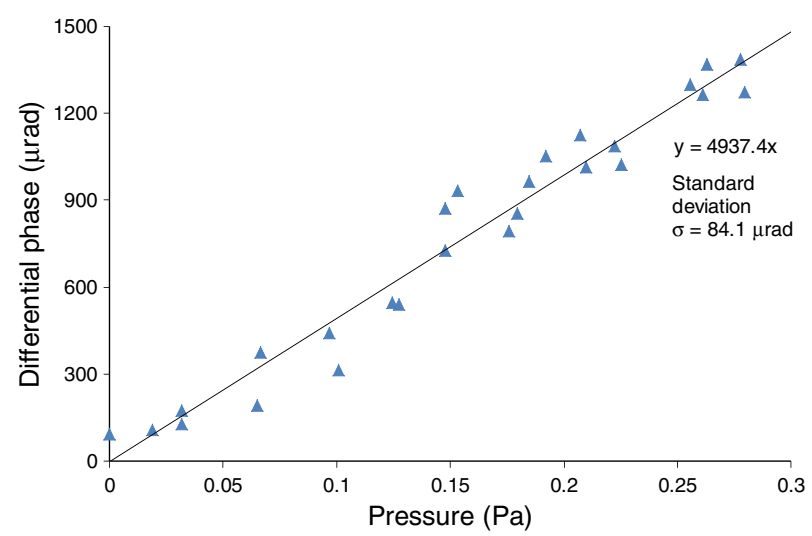

Fig. 3. (Color online) Measured relationship between differential phase $2 \Delta(\beta L)_{a}$ and acoustic pressure at a frequency of $70 \mathrm{~Hz}$, with linear fitting.

Assuming there is no coupling between the two orthogonal axes, the electric field amplitudes ( $\left.E_{\text {out }}\right)$ after returning through the PMC can be expressed as a matrix product of the initial electric field amplitudes $\left(E_{\text {in }}\right)$, the angle offset between the axes of the PMC and the OM $\left(R_{1,2}\right)$, the differential phase due to initial birefringence and acousto-optic effect $(P)$, and the inline mirror $(M)$ :

$$
E_{\text {out }}=R_{2} \cdot P \cdot M \cdot P \cdot R_{1} \cdot E_{\text {in }}
$$

The common time- and distance-dependent phase components are neglected as they have no impact on the BD output. The linearly polarized output light from the TLS is confined to one axis of the PM fiber:

$$
E_{\text {in }}=\left(\begin{array}{c}
E \\
0
\end{array}\right)
$$

The angle offset $(\alpha)$ between the axes of the PMC and the axes of the birefringent OM can be modeled by the rotation matrices:

$$
\begin{aligned}
& R_{1}=\left(\begin{array}{cc}
\cos \alpha & -\sin \alpha \\
\sin \alpha & \cos \alpha
\end{array}\right) \quad \text { and } \\
& R_{2}=\left(\begin{array}{cc}
\cos \alpha & \sin \alpha \\
-\sin \alpha & \cos \alpha
\end{array}\right)
\end{aligned}
$$

The differential phase $(\Delta \varphi)$ between the eigenmodes is governed by the initial birefringence $(\Delta \beta L)$ and the additional acoustic pressure-induced change in birefringence and fiber length $\left(\Delta(\beta L)_{a}\right)$ :

$$
\begin{gathered}
P=\left(\begin{array}{cc}
e^{i \phi} & 0 \\
0 & e^{-i \phi}
\end{array}\right), \\
\phi=\frac{\Delta \beta L+\Delta(\beta L)_{\mathrm{a}}}{2} .
\end{gathered}
$$

The in-line mirror can be modeled by:

$$
M=\left(\begin{array}{cc}
-1 & 0 \\
0 & 1
\end{array}\right)
$$


The electric field amplitudes at the PMC beat together and the resulting optical powers $\left(P_{\text {out }}\right)$ are a function of the angle offset and differential phase:

$$
\begin{aligned}
E_{\text {out }} & =\left(\begin{array}{c}
E_{x} \\
E_{y}
\end{array}\right) \\
& =E \cdot\left(\begin{array}{c}
-e^{2 i \phi} \cos ^{2} \alpha+e^{-2 i \phi} \sin ^{2} \alpha \\
e^{2 i \phi} \sin \alpha \cos \alpha+e^{-2 i \phi} \sin \alpha \cos \alpha
\end{array}\right), \\
& P_{\text {out }}=\left(\begin{array}{c}
P_{x} \\
P_{y}
\end{array}\right)=P \cdot\left(\begin{array}{c}
1-\sin ^{2}(2 \alpha) \cos ^{2}(2 \phi) \\
\sin ^{2}(2 \alpha) \cos ^{2}(2 \phi)
\end{array}\right) .
\end{aligned}
$$

The PC can be used to adjust the angle offset such that $\alpha=\pi / 4$, simplifying the BD output to:

$$
T=\frac{P_{x}-P_{y}}{P_{x}+P_{y}}=-\cos (4 \phi)=-\cos \left(2\left(\Delta \beta L+\Delta(\beta L)_{a}\right)\right) .
$$

Equation (9) can be written purely in terms of the acousto-optic contribution to the differential phase $\left(2 \Delta(\beta L)_{a}\right)$ provided that the initial birefringence-induced differential phase $(2 \beta L)$ is equal to $\pi / 2$ (plus integer multiples of $2 \pi$ ):

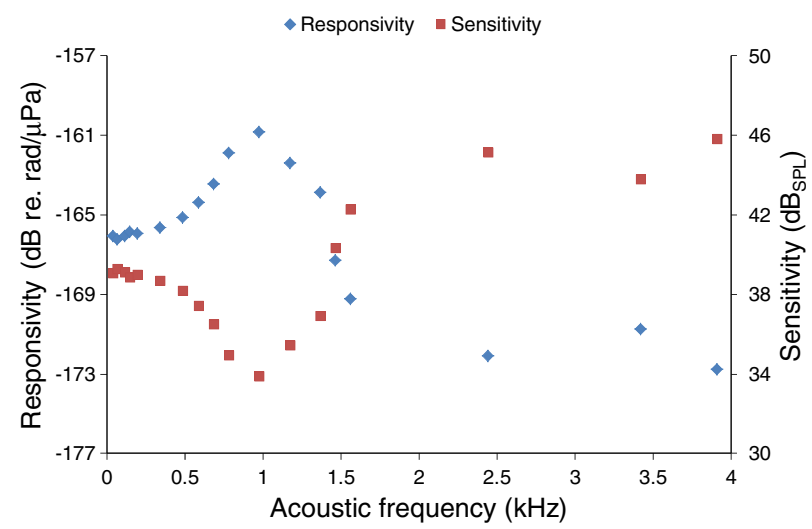

Fig. 4. (Color online) Measured acoustic frequency response and sensitivity.

$$
T=\sin \left(2 \Delta(\beta L)_{a}\right)
$$

The combination of the mirror reflection and balanced detection scheme $[\underline{7}, \underline{8}]$ suppresses common-mode noise from the TLS and doubles the phase-induced intensity modulation.

To characterize the birefringence of the fabricated sensor, light was launched through an inline polarizer aligned at $45^{\circ}$ to the $\mathrm{OM}$ followed by an analyzer set at $45^{\circ}$. An interferometric fringe period $(\Delta \lambda)$ of $61.93 \mathrm{~nm}$ was observed for a single pass through the sensor by sweeping the TLS wavelength. Hence, the quadrature con-dition in Eq. (무) can easily be achieved by tuning the input wavelength. This one-point calibration is to be performed without an acoustic signal before each measurement.

The BD (New Focus 2117) had a bandwidth of $10 \mathrm{kHz}$ and an overall response of $210 \mathrm{~V} / \mathrm{mW}$ for a gain setting of 300. Using Eq. (9), a total received power of $157.8 \mu \mathrm{W}$ corresponds to a maximum fringe amplitude of $33.14 \mathrm{~V}$. The measured fringe amplitude was $30.17 \mathrm{~V}$, indicating an interference visibility of 0.91 . The differential phase [Eqs. (9) and (10)] was calculated by dividing the output voltage of the $\mathrm{BD}$ by the measured fringe amplitude and performing an arcsine. A responsivity of $4.937 \mathrm{mrad}=\mathrm{Pa}$ shown in Fig. 3 for an acoustic signal of $70 \mathrm{~Hz}$ was calculated from dividing the differential phase by the acoustic pressure measured with the commercial microphone. 
The standard deviation is $84.1 \mu \mathrm{rad}$. The acoustic fre-quency response in Fig. 4 was obtained by measuring the responsivity at varying signal frequencies of fixed amplitude. The resonant frequency of the ABM was observed at $\sim 1 \mathrm{kHz}$. The average responsivity from 40 to $500 \mathrm{~Hz}$ and 1.5 to $4 \mathrm{kHz}$ are -166 and $-171 \mathrm{~dB} \mathrm{re} . \mathrm{rad} / \mu \mathrm{Pa}$, respectively.

Ambient acoustic noise was the dominant source of noise in the measurement data. Temperature drifts are not of great concern for acoustic signal frequencies above a few hertz, but acoustic noise ranged from a few hertz to a few kilohertz. Figure 5 shows an example measurement at $1562.5 \mathrm{~Hz}$ that was analyzed to check the ambient acoustic noise. The fast Fourier transform of the optical signal was derived and a signal-to-noise ratio of 11.6 was calcu-lated. This corresponds to a noise amplitude of $3.0 \mathrm{mV}$. The calculated noise-equivalent differential phase of $99.9 \mu \mathrm{rad}$ [Eqs. (9) and (10)] shows agreement with the $92.8 \mu \mathrm{rad}$ obtained without an acoustic signal in Fig. $\underline{3}$. The measured frequency-dependent responsivity was

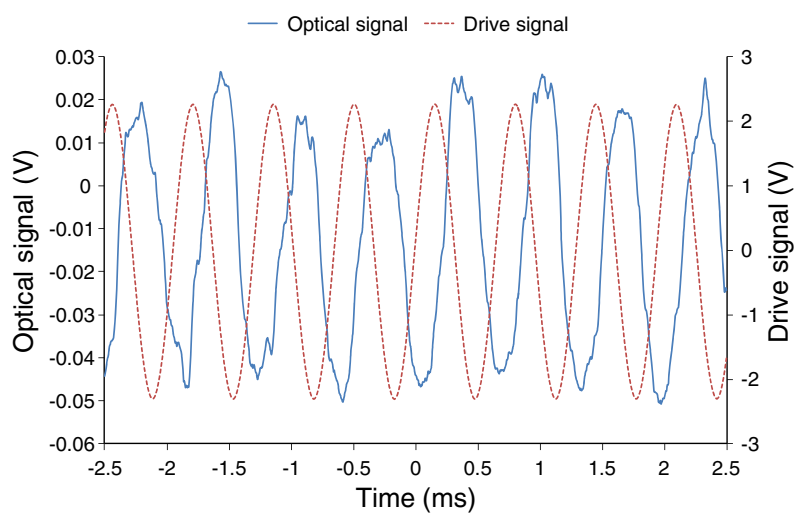

Fig. 5. (Color online) Optical signal and loudspeaker drive signal at a frequency of $1562.5 \mathrm{~Hz}$. The temporal offset is due to the time taken for acoustic pressure to reach the sensor.

$3.455 \mathrm{mrad} / \mathrm{Pa}$ or $\quad-169.2 \mathrm{~dB} \mathrm{re} . \mathrm{rad} / \mu \mathrm{Pa}$. Hence, the

ambient acoustic noise was $63.2 \mathrm{~dB}_{\mathrm{SPL}}($ re. $20 \mu \mathrm{Pa})$. The measured detector noise and shot noise were 353.5 and $149.2 \mu \mathrm{V}_{\text {rms }}$, respectively, yielding a phase sensitivity of $8.99 \mu \mathrm{rad}$ and an acoustic sensitivity of $42.3 \mathrm{~dB}_{\mathrm{SPL}}$ at the signal frequency of $1562.5 \mathrm{~Hz}$. The sensitivity is plotted as a function of frequency in Fig. 4 .

To optimize the acoustic responsivity, the length of the OM can be increased. Moreover, the wall thickness of the silicone rubber mandrel and the polymer packaging can be reduced to lower the stiffness while still maintaining clearance of the central support. To increase the reso-nant frequency and thus the operating bandwidth, a man-drel material of higher density and Young's modulus is ideal for the assembly of the ABM.

To reduce the optical loss, air bubbles must be removed from the polymer packaging.

In conclusion, we have demonstrated the use of OM for ABM-based acoustic sensors. The potential for high com-pactness and high responsivity is attractive in applica-tions with stringent space requirements, such as in defense and biomedical uses. A sensor using $2 \mu \mathrm{m}$ diam microfiber of $35 \mathrm{~mm}$ coiled length exhibited an average acoustic responsivity of -137 and $-142 \mathrm{~dB}$ re. $\mathrm{rad} / \mathrm{m} \cdot \mu \mathrm{Pa}$ from 40 to $500 \mathrm{~Hz}$ and 1.5 to $4 \mathrm{kHz}$, respectively. This is one order of magnitude higher than most conventional polarimetric ABM-based acoustic sensors [7], despite the miniaturization. The average acoustic sensitivity be-tween 40 and $500 \mathrm{~Hz}$ and 1.5 and $4 \mathrm{kHz}$ are 38.9 and 44.3 $\mathrm{dB}_{\mathrm{SPL}}$, respectively.

\section{References}

1. S. Niu, Y. Hu, Z. Hu, and H. Luo, IEEE Photon. Technol. Lett. 23, 1499 (2011).

2. H. Wagaard, G. B. Havsgård, and G. Wang, J. Lightwave Technol. 19, 994 (2001).

3. G. H. Ames and J. M. Maguire, J. Acoust. Soc. Am. 121, 1392 (2007).

4. Corning ClearCurve optical fiber datasheet, http://www .corning.com.

5. L. Tong, J. Lou, R. R. Gattass, S. He, X. Chen, L. Liu, and

E. Mazur, Nano Lett. 5, 259 (2005).

6. G. Brambilla, J. Opt. 12, 043001 (2010).

7. S. C. Rashleigh, Opt. Lett. 5, 392 (1980).

8. G. Y. Chen, T. Lee, R. Ismaeel, G. Brambilla, and T. P. Newson, IEEE Photon. Technol. Lett. 24, 860 (2012). 